\title{
Ökologische Grenzen des Handels?
}

\author{
Bisher hatte für die WTO Freihandel immer Vorrang gegenüber dem Schutz \\ von Umwelt und Gesundheit. Ein aktueller Schiedsspruch zu Hormonfleisch \\ deutet hier jedoch eine gewisse Trendwende an.
}

Von Susanne Dröge

$\mathrm{D}$ er seit 1989 währende Konflikt zwischen der Europäischen Union und den USA ist beigelegt. Der Urteilsspruch des Berufungsgerichts der Welthandelsorganisation (WTO) vom 16. Januar 1998 (1), lehnt zwar im Ergebnis das derzeitige EU-Importverbot von hormonbehandeltem Fleisch ab. Er beinhaltet jedoch einige Feststellungen, die eine neue Richtung im Umgang mit dem Schutz des Menschen und der Umwelt einschlagen. Bisher hatte sich die WTO in allen Streitfällen (z.B. im Thunfisch-Delphin-Streit zwischen USA und Mexiko) uneingeschränkt zugunsten des Freihandels ausgesprochen.

Das 1994 abgeschlossene Seitenabkommen des GATT (General Agreement on Tariffs and Trade) erlaubt den Mitgliedern, aufgrund nationaler Standards Handelsbeschränkungen für Produkte einzuführen, die Gesundheitsgefahren mit sich bringen. Allerdings sind dabei die Auflagen des WTO-Abkommens über sanitäre und phytosanitäre Standards (SPS) zu beachten, die zum ersten Mal in diesem Streitfall juristisch interpretiert worden sind. Der Panel - die erste Instanz, die von den WTOMitgliedern in Handelsstreitigkeiten angerufen werden kann - gab noch den USA recht, aber die Berufungsinstanz der WTO hat dem Panel in zwei von drei Punkten nicht oder nur teilweise zugestimmt.

Aus der Begründung sind folgende Einzelheiten für zukünftige Konflikte interessant. Erstens zieht entgegen dem Panel-Urteil die Anwendung eines höheren nationalen Gesundheitsstandards nicht gleichzeitig eine generelle Beweislast für das betreffende Land nach sich.

Zweitens werden die Vorschriften des SPS-Abkommens so ausgelegt, daß die Abweichung von internationalen Mindeststandards jedem Mitgliedsland zusteht, wenn es akute gesundheitliche Gefahren abwehren will. Wenn ein Land einen Standard einführen will, sollte es sich um Koordination und Harmonisierung mit anderen Staaten bemühen. Eine Harmonisierung der Schutzmaßnahmen aufgrund von internationalen Standards sei jedoch bisher noch nicht im Abkommen enthalten.
Drittens wird die Rolle der Risikobewertung geklärt, denn die USA hatten der EU vorgehalten, keine wissenschaftliche Begründung für das Importverbot vorgelegt zu haben. Grundsätzlich hielten die Berufungsrichter fest, daß jeder Schutzmaßnahme eine Risikobewertung zugrunde liegen muß, damit die abgeleiteten Handelsbeschränkungen von der WTO abgesegnet werden können. Was in diese Bewertung einfließen muß, wurde ebenfalls festgelegt. So umfaßt eine Risikobewertung nicht nur einen quantitativen Beweis einer gesundheitlichen Gefahr - wie er im Labor erfolgt -, sondern soll auch jegliche Risiken ,,der menschlichen Gesellschaft, wie sie in der Realität existieren", berücksichtigen. Risiken können auch neu auftreten und eine Beurteilung muß sich nach dem Potential der Gefährdung richten.

Allerdings liegt laut WTO für das Importverbot der EU noch keine entsprechende Risikobewertung vor. Offensichtlich mangelt es den von der EU angeführten Studien an dem speziellen Nachweis, daß das mit Wachstumshormonen hergestellte Fleisch Krebs erregen kann.

Für die zukünftigen Streitfälle vor der WTO wird das Urteil Veränderungen bringen. Zum ersten Mal hat eine WTO-Instanz sich mit den komplexen Zusammenhängen von Gesundheitsrisiken und Produktionsmethoden befaßt. Sie hat den Begriff des Risikos definiert und dabei insbesondere den Schutz des Menschen und der Umwelt hervorgehoben. Mit der Forderung nach Risikobewertungen als Grundlage für nationale Handelsbeschränkungen hat sie klare Auflagen erteilt. Diese Aspekte lassen den Schluß zu, daß die Interessen des Gesundheits- und Umweltschutzes jetzt auch bei den Vertretern des freien Welthandels auf Gehör gestoßen sind.

Anmerkung

(1) Das Urteil ist über http://www.wto.org abrufbar.

\section{Die Autorin}

Susanne Dröge ist wiss. Mitarbeiterin am Lehrstuhl für Makroökonomie der Handelshochschule Leipzig (HHL).

Kontakt: HHL, Jahnallee 59, Tel. 0341/ 98 51-657,

E-mail: droege@macroec.hhl.de
Zum Thema ...

Sechsmal im Jahr: Die Fachzeitschrift für Umweltrecht -

und damit sechsmal im Jahr ein kompletter Überblick über das Umweltrecht.

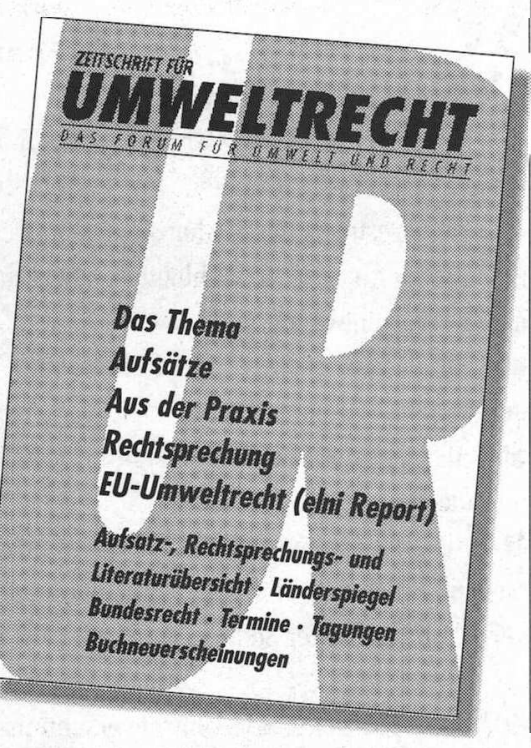

\section{Aktuelle wissenschaftliche Beiträge und Analysen}

- diskutieren Stand und Entwicklung des Umweltrechts.

\section{Ein umfangreicher Service-Teil}

- bringt die neueste Rechtsprechung,

- informiert über die aktuelle Gesetzesentwicklung auf Landes-, Bundes- und Europaebene und

- dokumentiert Aufsätze aus über einhundert Fachzeitschriften sowie wichtige Nachrichten, Termine und Buchneuerscheinungen.

Zu bestellen bei: Rhombos-Verlag

Kurfürstenstr. 17, 10785 Berlin

Tel. 030/2619461, Fax 030/2616854 
(c) 20I0 Authors; licensee IÖW and oekom verlag. This is an article distributed under the terms of the Creative Commons Attribution Non-Commercial No Derivates License (http://creativecommons.org/licenses/by-nc-nd/3.o/), which permits unrestricted use, distribution, and reproduction in any medium, provided the original work is properly cited. 